\title{
Scalar mode propagation in modified gravity with a scalar field
}

\author{
Antonio De Felice* and Teruaki Suyamat \\ Theoretical and Mathematical Physics Group, Centre for Particle Physics and Phenomenology, \\ Louvain University, 2 Chemin du Cyclotron, 1348 Louvain-la-Neuve (Belgium)
}

(Dated: November 11, 2018)

\begin{abstract}
We study the propagation of the scalar modes around a Friedmann-Lemaître-Robertson-Walker universe for general modifications of gravity in the presence of a real scalar field. In general, there will be two propagating scalar perturbation fields, which will have in total four degrees of freedom. Two of these degrees will have a superluminal propagation - with $k$-dependent speed of propagationwhereas the other two will have the speed of light. Therefore, the scalar degrees of freedom do not modify the general feature of modified gravity models: the appearance of modes whose frequency depends on the second power of the modulus of the wave vector. Constraints are given and special cases are discussed.
\end{abstract}

\section{INTRODUCTION}

In [1], the scalar cosmological perturbation theory around a general Friedmann-Lemaître-Robertson-Walker (FLRW)

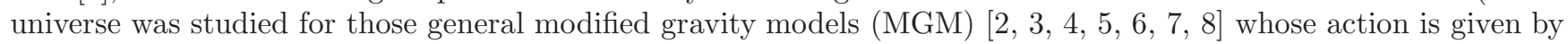

$$
S=\frac{M_{P}^{2}}{16 \pi} \int \mathrm{d}^{4} x \sqrt{-g} f(R, \mathcal{G}),
$$

where $f(R, \mathcal{G})$ is a general function, $R$ is Ricci scalar, and $\mathcal{G}$ is the so-called Gauss-Bonnet term defined by

$$
\mathcal{G} \equiv R^{2}-4 R_{\mu \nu} R^{\mu \nu}+R_{\mu \nu \alpha \beta} R^{\mu \nu \alpha \beta} .
$$

It was found that, in general, the propagation equation for the metric perturbation was acquiring a term proportional to $k^{4}$, where $k$ is the length of the comoving wavenumber vector $k \equiv|\vec{k}|$. This $k^{4}$-term implies that, depending on the sign of the coefficient in front of the $k^{4}$-term, either the universe is very unstable on small scales or the short wavelength fluctuations propagate with speed larger than the speed of light. The background classical-instabilities, due to the presence of a negative $k^{4}$-term, must be forbidden, whereas there still exists controversy as to if the superluminal propagation is compatible or not with experimental observations [9, 10, 11, 12, 13]. Anyhow, the scalar cosmological perturbation for the general MGM exhibits highly non-trivial and interesting features that can be used to set constraints on the MGM or may even leave detectable imprints on cosmic structures due to the $k^{4}$-term.

These results in [1] were found for the vacuum only, i.e. no fields other than the gravitational field were included in the Lagrangian. An obvious and important extension of this work is to add some matter fields into the MGM action and to see how the results we found in the vacuum case, are affected by the existence of matter. This is the main purpose of this paper. As a first and simple extension, although useful in early-times cosmology, in this paper, we will consider a general MGM together with a single real scalar field $\varphi$, whose action is given by

$$
S=\frac{M_{P}^{2}}{16 \pi} \int \mathrm{d}^{4} x \sqrt{-g} f(R, \mathcal{G}, \varphi)-\frac{1}{2} \int \mathrm{d}^{4} x \sqrt{-g} w \partial^{\mu} \varphi \partial_{\mu} \varphi .
$$

If $w$ depends on $\varphi$, we can set $w$ to be a constant by a suitable field redefinition. Therefore, we will consider $w$ as a constant from the beginning. In this setup, when $w=0$, the Lagrangian reduces to the model in vacuum (11) ${ }^{1}$. We can even remove $w$, if $w>0$, by redefining the scalar field as $\tilde{\varphi}=\sqrt{w} \varphi$. However, we will leave $w$ as a free parameter to enable us to consider those cases for which $w<0$. We do not assume any particular functional form for $f(R, \mathcal{G}, \varphi)$.

*Electronic address: antonio.defelice@uclouvain.be

$\dagger$ Electronic address: teruaki.suyama@uclouvain.be

${ }^{1}$ When $w=0, \varphi$ becomes an auxiliary field. From a variation with respect to $\varphi$, we obtain $\varphi$ as a function of $R$ and $\mathcal{G}$, i.e. $\varphi=\varphi(R, \mathcal{G})$. Removing $\varphi$ in the action by using this relation yields an action which only contains $R$ and $\mathcal{G}$. Therefore, obtained action belongs to (11). 
Although (3) is the basic action we consider, for convenience of the actual analysis, we mainly use a different action, equivalent to (3), which is given by

$$
\begin{aligned}
S & =\frac{M_{P}^{2}}{16 \pi} \int \mathrm{d}^{4} x \sqrt{-g}[f(\lambda, \sigma, \varphi)+(R-\lambda) F(\lambda, \sigma, \varphi)+(\mathcal{G}-\sigma) \xi(\lambda, \sigma, \varphi)]-\frac{1}{2} \int \mathrm{d}^{4} x \sqrt{-g} w \partial^{\mu} \varphi \partial_{\mu} \varphi \\
& =\frac{M_{P}^{2}}{16 \pi} \int \mathrm{d}^{4} x \sqrt{-g}[R F(\lambda, \sigma, \varphi)+\mathcal{G} \xi(\lambda, \sigma, \varphi)-V(\lambda, \sigma, \varphi)]-\frac{1}{2} \int \mathrm{d}^{4} x \sqrt{-g} w \partial^{\mu} \varphi \partial_{\mu} \varphi
\end{aligned}
$$

where $\lambda$ and $\sigma$ are auxiliary fields and

$$
\begin{aligned}
F(\lambda, \sigma, \varphi) & \equiv \frac{\partial f}{\partial \lambda}, \\
\xi(\lambda, \sigma, \varphi) & \equiv \frac{\partial f}{\partial \sigma}, \\
V(\lambda, \sigma, \varphi) & \equiv \lambda F(\lambda, \sigma, \varphi)+\sigma \xi(\lambda, \sigma, \varphi)-f(\lambda, \sigma, \varphi) .
\end{aligned}
$$

By the following way, we can verify that the action (4) is equivalent to (3). By variating $S$ with respect to $\lambda$ and $\sigma$, we have the equations for $\lambda$ and $\sigma$, which are given by

$$
\begin{aligned}
& (R-\lambda) F_{\lambda}+(\mathcal{G}-\sigma) F_{\sigma}=0 \\
& (R-\lambda) F_{\sigma}+(\mathcal{G}-\sigma) \xi_{\sigma}=0
\end{aligned}
$$

where $F_{\lambda}=\partial F / \partial \lambda$ etc. If the combination $F_{\lambda} \xi_{\sigma}-F_{\sigma}^{2}$ does not vanish, the two equations are independent and $\lambda$ and $\sigma$ are given by

$$
\begin{aligned}
\lambda & =R, \\
\sigma & =\mathcal{G} .
\end{aligned}
$$

Eliminating $\lambda$ and $\sigma$ in the original action by using these results, we find that $S$ in (4) reduces to (3): the equivalence of these two actions also holds for the equations of motion. If the combination $F_{\lambda} \xi_{\sigma}-F_{\sigma}^{2}$ vanishes, (7) and (8) are not independent from each other. This does not mean we can not eliminate $\lambda$ and $\sigma$ from the original action. Because (7) is the condition that the action remains the same under the variation, all the possible pairs of $\lambda$ and $\sigma$ that satisfy (17) give the same action. We can put any $(\lambda, \sigma)$ we want into $S$ as long as $(\lambda, \sigma)$ are the solutions of (7). Obviously, $\lambda=R$ and $\sigma=\mathcal{G}$ are the solution and we can put them into (4) to find that it recovers the original action (3).

In the next section, we take the action approach to study the cosmological scalar perturbations. That is, we will expand the action (4) to second order in the perturbation and reveal new propagation properties due to the inclusion of the scalar field. After the classical analysis of the perturbations, we will also consider quantization of the perturbations.

\section{ACTION}

\section{A. Background and Perturbation Variable}

We consider a spacetime which slightly deviates from a FLRW universe. We write the metric as

$$
\mathrm{d} s^{2}=-(1+2 \alpha) \mathrm{d} t^{2}-2 a(t) \partial_{i} \beta \mathrm{d} t \mathrm{~d} x^{i}+a^{2}(t)\left(\delta_{i j}+2 \phi \delta_{i j}+2 \partial_{i} \partial_{j} \gamma\right) \mathrm{d} x^{i} \mathrm{~d} x^{j},
$$

where $a(t)$ is the scale factor and $\alpha, \beta, \phi$ and $\gamma$ represent the metric perturbations. We only consider the scalar type perturbation, as both the vector and tensor modes' propagation will not be affected-at linear order-by the presence of the scalar field, albeit the modified background dynamical evolution. We also decompose the scalar field as $\varphi+\delta \varphi$, where $\varphi$ is the background value and $\delta \varphi$ is the perturbation. Since $f$ is a function of $R$ and $\mathcal{G}$, which must be expanded, $F$ and $\xi$ defined by (5) also acquire perturbations, which we denote as $\delta F$ and $\delta \xi$. For later convenience, we define $Z$ and $\chi$ by the following equation,

$$
Z \equiv \frac{\partial f}{\partial \varphi}, \quad \chi \equiv a(\beta+a \dot{\gamma})
$$

There are degrees of freedom of choosing $\Sigma_{t}$ which is a three dimensional hyper-surface of constant $t$. Changing from $\Sigma_{t}$ to $\tilde{\Sigma}_{t}$ corresponds to a time coordinate transformation: $t \rightarrow t+T\left(t, x^{i}\right)$. Under this transformation, the 
perturbation variables transform as

$$
\begin{aligned}
\tilde{\alpha} & =\alpha-\dot{T}, \\
\tilde{\phi} & =\phi-H T, \\
\tilde{\chi} & =\chi-T, \\
\tilde{\delta \varphi} & =\delta \varphi-\dot{\varphi} T, \\
\tilde{\delta F} & =\delta F-\dot{F} T, \\
\tilde{\delta \xi} & =\delta \xi-\dot{\xi} T, \\
\tilde{\delta Z} & =\delta Z-\dot{Z} T .
\end{aligned}
$$

\section{B. First order action}

To obtain the background equations of motion, we need to expand the action (4) at first order in the perturbation variables. We find that, at first order, the action is given by

$$
\begin{aligned}
S^{(1)}=\frac{M_{p}^{2}}{16 \pi} \int \mathrm{d}^{3} x \mathrm{~d} t a^{3}[ & 3\left(-V+6 \dot{H} F+12 H^{2} F+24 H^{4} \xi+24 H^{2} \dot{H} \xi\right) \phi+24 H\left(F+4 H^{2} \xi+2 H \dot{H} \xi\right) \dot{\phi} \\
& +6\left(F+4 H^{2} \xi\right) \ddot{\phi}-\left(V+6 \dot{H} F+12 H^{2} F+72 H^{2} \dot{H} \xi+72 H^{4} \xi\right) \alpha \\
& \left.-6 H\left(F+4 H^{2} \xi\right) \dot{\alpha}+Z \delta \varphi\right]+\int \mathrm{d}^{3} x \mathrm{~d} t a^{3}\left[\frac{w}{2} \dot{\varphi}^{2}(\alpha+3 \phi)+w \dot{\varphi} \dot{\delta}\right] .
\end{aligned}
$$

The variation with respect to the auxiliary fields $\delta \lambda$ and $\delta \sigma$ can also contribute to $S^{(1)}$. It gives the background equations $\lambda=R$ and $\sigma=G$. In (20), we have already replaced $\lambda, \sigma$ by $R, G$ respectively and eliminated terms proportional to $\delta \lambda$ and $\delta \sigma$.

By integrating by parts, we find that the condition $S^{(1)}=0$ yields the background equations which are given by

$$
\begin{aligned}
3 H^{2} & =\frac{1}{F}\left(\frac{1}{2} V-3 H \dot{F}-12 H^{3} \dot{\xi}+\frac{4 \pi w}{M_{P}^{2}} \dot{\varphi}^{2}\right), \\
\dot{H} & =\frac{1}{2 F+8 H \dot{\xi}}\left[-\ddot{F}+H \dot{F}-4 H^{2}(\ddot{\xi}-H \dot{\xi})-\frac{8 \pi w}{M_{P}^{2}} \dot{\varphi}^{2}\right], \\
w \ddot{\varphi} & +3 w H \dot{\varphi}-\frac{M_{P}^{2}}{16 \pi} Z=0 .
\end{aligned}
$$

\section{Second order action}

To obtain the perturbation equations, we need to expand the action up to second order in the perturbation variables. We denote the second order action as $S^{(2)}$. Although the calculation is straightforward, the expressions at each step of the algebraic calculation become very long. Therefore we shall be content with explaining what we do at each calculation step and giving the final expression of the second order action which contains only dynamical fields.

The field variables we can perturb as free variables are $\alpha, \chi, \phi, \delta \varphi, \delta F$ and $\delta \xi$. Note that $\delta \lambda$ and $\delta \sigma$ can be written as linear combinations of $\delta F, \delta \xi$ and $\delta \varphi$ by inverting the relations

$$
\begin{aligned}
\delta F & =F_{\lambda} \delta \lambda+F_{\sigma} \delta \sigma+F_{\varphi} \delta \varphi, \\
\delta \xi & =F_{\sigma} \delta \lambda+\xi_{\sigma} \delta \sigma+\xi_{\varphi} \delta \varphi .
\end{aligned}
$$

Just for practical convenience, we will use $\delta F$ and $\delta \xi$ instead of $\delta \lambda$ and $\delta \sigma$.

By using those fields $(\alpha, \chi, \phi, \delta \varphi, \delta F, \delta \xi)$, we can expand the action up to second order in those fields. Next, we chose a suitable gauge to make the calculation easier, the so-called Modified Gravity Models Gauge (MGMG), where $\delta F+4 H^{2} \delta \xi=0$. This gauge condition uniquely fixes the time slicing. By this gauge condition, we can remove $\delta F$ from $S^{(2)}$, so that $S^{(2)}$ is a quadratic functional of $\left\{\alpha_{M}, \chi_{M}, \phi_{M}, \delta \varphi_{M}, \delta \xi_{M}\right\}$, where the index $M$ indicates that we are working in the MGMG. After a few integration by parts, we find that $\alpha_{M}, \chi_{M}$ and $\delta \xi_{M}$ are auxiliary fields. 
Eliminating these fields by using their equations of motion, $S^{(2)}$ becomes a functional of $\phi_{M}$ and $\delta \varphi_{M}$ only. Performing other integrations by parts, we find that $S^{(2)}$ can be finally written as

$$
\begin{gathered}
S^{(2)}=\frac{M_{p}^{2}}{16 \pi} \int \mathrm{d} t \mathrm{~d}^{3} \vec{x} a^{3}(t)\left[A_{a b}(t) \dot{V}_{a} \dot{V}_{b}+a(t)^{-2} B(t) \epsilon_{a b} \vec{\nabla} \dot{V}_{a} \cdot \vec{\nabla} V_{b}-a(t)^{-4} D_{a b}(t) \triangle V_{a} \Delta V_{b}-a(t)^{-2} E_{a b}(t) \vec{\nabla} V_{a} \cdot \vec{\nabla} V_{b}\right. \\
\left.+C(t) \epsilon_{a b} \dot{V}_{a} V_{b}+M_{a b}(t) V_{a} V_{b}\right]
\end{gathered}
$$

Here $V_{a}$ with $(a=1,2)$ is defined as

$$
V_{1}=\phi_{M}, \quad V_{2}=\delta \varphi_{M}
$$

The matrices $A, D, E$ and $M$ are given by background quantities. The matrix $\epsilon_{a b}$ is the pure antisymmetric matrix with element $\epsilon_{12}=1$. Furthermore, the only non-zero matrix element for $D$ is $D_{11}$. In the short wavelength regime, $A, B, D$ and $E$ alone determine the propagation nature of the perturbations. Explicit expressions of these matrices are given in the appendix.

From this action, we can derive some important results regarding the propagation properties of the short wavelength modes. First, the determinant of $A$ does not vanish in general. Therefore, there are two independent dynamical perturbation fields (hence four degrees of freedom). The reason why we get two dynamical fields is clear. In the absence of the scalar field, i.e. in vacuum, it was found that there is only one dynamical field coming from the metric perturbation. Because we are now adding a dynamical scalar field which has nothing to do with the gravitational sector, it is not surprising that this scalar field brings new degrees of freedom. As an obvious extension, we will get $2(N+1)$ degrees of freedom for the scalar perturbations if we add $N$ dynamical and independent scalar fields into the action.

In order not to have ghosts, we require that the two eigenvalues of $A_{a b}$ must be positive. This condition is equivalent to the following conditions,

$$
\operatorname{det} A>0, \quad \operatorname{tr} A>0 \text {. }
$$

Second important result is that $D$ is not a zero matrix in general. Therefore, the $k^{4}$-term which has already been present in the vacuum case is still present even if the scalar field is added into the theory. Explicit calculations show that only $D_{11}$ does not vanish. Therefore, among the two dynamical fields, only one acquires $k^{4}$-propagation. This, we think, is the most important contribution of this paper. In fact, since $V_{1}$ represents the metric and $V_{2}$ describes the perturbation of the scalar field, we can say that only the metric perturbation acquires the $k^{4}$-term behaviour. We can understand this result as follows. Since the $k^{4}$-term is already present in the vacuum case, the appearance of the $k^{4}$-term is solely related to the nature of the modification of gravity. As for the scalar fields, it has a standard kinetic term and it is hard to imagine that it would give an additional $k^{4}$-term contribution from its kinetic term. In fact, we find that $D_{11}$ is proportional to the combination $F_{\lambda} \xi_{\sigma}-F_{\sigma}^{2}$ (see appendix), which distinguishes classes of modifications of gravity. Therefore, $F_{\lambda} \xi_{\sigma}-F_{\sigma}^{2}=0$ is still applicable as a sufficient condition for the absence of the $k^{4}$ propagation. Below, we will consider a general case where $F_{\lambda} \xi_{\sigma}-F_{\sigma}^{2} \neq 0$, whereas, in the next section, we will consider some special case where, for example, $F_{\lambda} \xi_{\sigma}-F_{\sigma}^{2}=0$.

From (26), we can derive the equations of motion for $V_{a}$. Using a Fourier transformation, the equations of motions for the two modes are

$$
\frac{\left(a^{3} A_{a b} \dot{V}_{b}\right)}{a^{3}}+\frac{k^{2}}{2 a^{5}}\left(a^{3} B \epsilon_{a b} V_{b}\right)+\frac{k^{2}}{2 a^{2}} B \epsilon_{a b} \dot{V}_{b}+D_{a b} \frac{k^{4}}{a^{4}} V_{b}+E_{a b} \frac{k^{2}}{a^{2}} V_{b}+\frac{\left(a^{3} C \epsilon_{a b} V_{b}\right)}{2 a^{3}}+\frac{1}{2} C \epsilon_{a b} \dot{V}_{b}-M_{a b} V_{b}=0
$$

In the short wavelength limit ( $k$ large), this vectorial equation can be approximated as

$$
A_{a b} \ddot{V}_{b}+\frac{k^{2}}{a^{2}} B \epsilon_{a b} \dot{V}_{b}+D_{a b} \frac{k^{4}}{a^{4}} V_{b}+E_{a b} \frac{k^{2}}{a^{2}} V_{b}=0
$$

From this equation, we can derive a dispersion relation. By denoting the angular frequency for the mode $k$ as $\omega$, the dispersion relation is given by

$$
\operatorname{det}\left(-\omega^{2} A+i \omega \frac{k^{2}}{a^{2}} B \epsilon+\frac{k^{4}}{a^{4}} D+\frac{k^{2}}{a^{2}} E\right)=0
$$

This is a quadratic equation in $\omega^{2}$ and it has two solutions. As we discussed before, only two modes among four acquire $k^{4}$ propagation. From (31), to leading order in $k$, we find that the angular frequency for those modes can be written as

$$
\omega^{2} \approx \frac{B^{2}+\operatorname{tr} A \operatorname{tr} D-\operatorname{tr}(A D)}{\operatorname{det} A} \frac{k^{4}}{a^{4}}=\frac{B^{2}+A_{22} D_{11}}{A_{11} A_{22}-A_{12}^{2}} \frac{k^{4}}{a^{4}}
$$


Using the explicit expression for the matrix elements given in the appendix, $\omega^{2}$ is given by

$$
\omega^{2}=-\frac{64}{3} \frac{\dot{H}^{2}\left(F_{\lambda} \xi_{\sigma}-F_{\sigma}^{2}\right)}{(F+4 H \dot{\xi})\left(F_{\lambda}+8 H^{2} F_{\sigma}+16 H^{4} \xi_{\sigma}\right)} \frac{k^{4}}{a^{4}} .
$$

It is impressive that this expression, in form, is exactly the same as that in the vacuum case [1]. However, by using (24), we can replace, for example, $F_{\lambda}$ with different background quantities such as $\dot{F}, F_{\sigma}, F_{\varphi}$ and $\dot{\varphi}$. After this replacement, $\omega$ explicitly depends on the scalar fields and is not anymore the same as the vacuum case. There is no clear distinction between implicit and explicit dependence of the scalar field on $\omega^{2}$. To conclude, the introduction of the scalar field does not remove the $k^{4}$ propagation but changes the background dependent coefficients in the dispersion relation.

As discussed in detail in [1], if the right hand side of (33) is negative, the perturbation grows exponentially in time. The growth rate is higher for shorter wavelength (proportional to $k^{2}$ ). Therefore, the FLRW universe is very unstable. If the RHS is positive, the perturbation propagate as a wave. In this case, the propagation speed (group velocity) is linearly dependent on $k$. Therefore, the propagation speed is in general superluminal at small scales.

As for the dispersion relation for the other two modes, we find that $\omega$, to leading order in $k$, can written as

$$
\omega^{2}=\frac{D_{11} E_{22}}{B^{2}+A_{22} D_{11}} \frac{k^{2}}{a^{2}}=\frac{k^{2}}{a^{2}} .
$$

Therefore, these modes propagate at the speed of light.

\section{SPECIAL CASES}

In the previous section, we have found that the scalar perturbations have four degrees of freedom (two dynamical fields). Among them, two modes acquire a $k^{4}$ propagation which depends on background quantities, whereas the other two propagate at the speed of light. However, there are special cases for which we can not apply this generic analysis. This is the case if $f(R, \mathcal{G}, \varphi)$ satisfies the special relation $F_{\lambda} \xi_{\sigma}-F_{\sigma}^{2}=0$, or $w=0$, or the universe undergoes de-Sitter expansion. In the following, we will consider each case separately.

\section{A. Case I: $F_{\lambda} \xi_{\sigma}-F_{\sigma}^{2}=0, w \neq 0$}

In this case, both $B$ and $D_{a b}$ vanish identically. Therefore, the dispersion relation (31) simplifies to

$$
\operatorname{det}\left(-\omega^{2} A+\frac{k^{2}}{a^{2}} E\right)=0 .
$$

Explicit forms of the matrix elements of $A$ and $E$ are given in the appendix. This equation yields a quartic equation for $\omega$, which is given by

$$
\operatorname{det} A \omega^{4}-\frac{k^{2}}{a^{2}}[\operatorname{tr} A \operatorname{tr} E-\operatorname{tr}(A E)] \omega^{2}+\frac{k^{4}}{a^{4}} \operatorname{det} E=0 .
$$

This equation always has real solutions for $\omega^{2}$. Explicit forms of $\operatorname{det} A, \operatorname{det} E$ and $\operatorname{tr} A \operatorname{tr} E-\operatorname{tr}(A E)$ in this case are also given in the appendix.

Eq. (36) has two different solutions for $\omega^{2}$, in general. Interestingly $\omega^{2}=k^{2} / a^{2}$ is not generally a solution of (36) any longer. This can be seen by substituting this ansatz into (36). Under this substitution, the LHS of (36) becomes

$$
\frac{k^{4}}{a^{4}}-\frac{k^{4}}{a^{4}} \frac{\operatorname{tr} A \operatorname{tr} E-\operatorname{tr}(A E)}{\operatorname{det} A}+\frac{k^{4}}{a^{4}} \frac{\operatorname{det} E}{\operatorname{det} A}=-\frac{4}{3} \frac{M_{P}^{2} \dot{H}^{2}\left(\dot{F} \xi_{\sigma}-\dot{\xi} F_{\sigma}\right)^{2}}{\pi w \dot{\varphi}^{2}(F+4 H \dot{\xi})\left(F_{\sigma}+4 H^{2} \xi_{\sigma}\right)^{2}} \frac{k^{4}}{a^{4}} .
$$

Assuming the universe is not de Sitter (the de Sitter case will be studied as another special case), this does not vanish unless $\dot{F} \xi_{\sigma}-\dot{\xi} F_{\sigma}=0$. This happens, for example, when $f=f(R, \mathcal{G})-U(\varphi)$.

If at least one of the solutions for $\omega^{2}$ is negative, the perturbations are classically unstable. The condition for not having ghosts modes (28), and the condition that the two $\omega^{2}$ are both positive are equivalent to

$$
\operatorname{tr} A>0, \quad \operatorname{det} A>0, \quad \operatorname{det} E>0, \quad \operatorname{tr} A \operatorname{tr} E-\operatorname{tr}(A E)>0 .
$$


Just for the purpose of seeing how our findings are applied to some concrete models, let us consider simple examples which have been frequently studied in literature. The first one is [14, 15, 16]

$$
f(R, \mathcal{G}, \varphi)=f(R, \varphi) .
$$

In this case, we have $\dot{\xi}=\xi_{\sigma}=F_{\sigma}=0$ and

$$
\operatorname{det} A=\frac{3 \frac{16 \pi}{M_{P}^{2}} w F \dot{F}^{2}}{(\dot{F}+2 H F)^{2}}, \quad \operatorname{tr} A \operatorname{tr} E-\operatorname{tr}(A E)=2 \operatorname{det} A, \quad \operatorname{det} E=\operatorname{det} A .
$$

Therefore, the dispersion relation becomes

$$
\operatorname{det} A\left(\omega^{2}-\frac{k^{2}}{a^{2}}\right)^{2}=0
$$

Therefore, assuming $\operatorname{det} A \neq 0$, all the four modes propagate at the speed of light. There are, however, well-known exceptions, i.e. GR and the Brans-Dicke theory. In GR, $F=1$ and $\dot{F}=0$ and hence $\operatorname{det} A=0$. This means that one of the fields does not propagate. The perturbation fields in (26) are defined on MGMG. In GR, this gauge condition is automatically satisfied, so that there still remains some gauge degrees of freedom. By using this remaining gauge, we can always set one of the two perturbation fields to be zero.

For the Brans-Dicke theory, $f(R, \mathcal{G}, \varphi)$ is given by

$$
f(R, \mathcal{G}, \varphi)=\frac{2 \pi}{M_{P}^{2}} \varphi^{2} R
$$

Using a new field defined by $\psi=\varphi^{2} / 8$, the action indeed reduces to the original one proposed in [17],

$$
S=\int \mathrm{d}^{4} x \sqrt{-g}\left(\psi R-\frac{w}{\psi} \partial^{\mu} \psi \partial_{\mu} \psi\right) .
$$

Therefore, for Brans-Dicke theory, $F=2 \pi \varphi^{2} / M_{P}^{2}$ and $\xi=0$. Then MGMG imposes that $V_{2}=\delta \varphi_{M}=0$. Therefore, only the $V_{1}$ field is dynamical and $A_{11}$ and $E_{11}$ determine alone the propagation nature of the perturbations. Applying the explicit expressions for $A_{11}$ given in the appendix, we find that $A_{11}$ is given by

$$
A_{11}=(2 w+3) \frac{\varphi^{2} \dot{\varphi}^{2}}{4(\dot{\varphi}+H \varphi)^{2}} .
$$

Therefore, a ghost appears when $w<-3 / 2$, which agrees with well known results [18]. Furthermore, it can be shown that the propagation speed of the perturbations is the speed of light.

Another similar case is the Lagrangian with

$$
f(R, \mathcal{G}, \varphi)=F(\varphi) R+\xi(\varphi) \mathcal{G}-U(\varphi), \quad \text { and } \quad w \neq 0 .
$$

In this case the number of propagating fields is only one, as the MGMG corresponds to setting $\delta \varphi=0$. This case was the only one studied in [19], although the authors claimed to have studied $f(R, \mathcal{G}, \varphi)=f_{1}(\varphi, R)+\xi(\varphi) \mathcal{G}-U(\varphi)$, with general $f_{1}$, which has, instead, two propagating fields.

A second example for a special case is given by [20, 21],

$$
f(R, \mathcal{G}, \varphi)=R+f_{1}(\mathcal{G}, \varphi)
$$

In this case, we have $F=1, \dot{F}=0$ and

$$
\begin{aligned}
\operatorname{det} A & =\frac{12 H^{2} \dot{\xi}^{2} \frac{16 \pi}{M_{P}^{2}} w(1+4 H \dot{\xi})}{(1+6 H \dot{\xi})^{2}}, \\
\operatorname{tr} A \operatorname{tr} E-\operatorname{tr}(A E) & =\frac{8 \frac{16 \pi}{M_{P}^{2}} w \dot{\xi}^{2}}{(1+6 H \dot{\xi})^{2}}\left(3 H^{2}+14 H^{3} \dot{\xi}+2 \dot{H}-2 H^{2} \ddot{\xi}+8 H \dot{H} \dot{\xi}\right), \\
\operatorname{det} E & =\frac{16 \pi}{M_{P}^{2}} w \frac{4 \dot{\xi}^{2}}{(1+6 H \dot{\xi})^{2}}\left(16 H\left(H^{2}+\dot{H}\right) \dot{\xi}-4 H^{2} \ddot{\xi}+3 H^{2}+4 \dot{H}\right) .
\end{aligned}
$$


Then the dispersion relation becomes

$$
\left(\omega^{2}-\frac{k^{2}}{a^{2}}\right)\left[\omega^{2}-\left(1+\frac{2 \dot{H}}{H^{2}}+\frac{\frac{16 \pi}{M_{P}^{2}} w}{(1+4 H \dot{\xi}) H^{2}} \dot{\varphi}^{2}\right) \frac{k^{2}}{a^{2}}\right]=0 .
$$

We find that two out of four modes propagate at the speed of light. This result is consistent with the discussion we did just after (37). As for the other two modes, the square of the propagation speed is

$$
c_{s}^{2}=1+\frac{2 \dot{H}}{H^{2}}+\frac{16 \pi w}{M_{P}^{2}(1+4 H \dot{\xi}) H^{2}} \dot{\varphi}^{2} .
$$

In vacuum, we would get $1+2 \dot{H} / H^{2}$ which agrees with [1]. There, it was found that the universe must be accelerating in order to avoid a negative $c_{s}^{2}$, furthermore the propagation was becoming superluminal during super-acceleration, i.e. $\dot{H}>0$. In the presence of a scalar field, (51) implies that, depending on the sign of $w(1+4 H \dot{\xi})$, the scalar field contribution can shift $c_{s}^{2}$ toward either positive or negative values. Therefore, in principle, it is possible to have positive and sub-luminal $c_{s}^{2}$ even in a decelerating universe.

\section{B. Case II: non-propagating field $-w=0$}

Let us here discuss the case in which $w=0$. This is a special case, which can be understood by noticing that in this case $\varphi$ becomes an auxiliary field. In fact, after integrating it out, one can show that the Lagrangian reduces to the vacuum $f(R, \mathcal{G})$ case already discussed in [1]. However it is also possible to keep the field $\varphi$, and expand the action at second order in all the fields. In this case, choosing the MGMG gauge, one finds only one propagating field, $\phi_{M}$, as expected. However, the $k^{4}$-term is still present, and one finds that for large $k$, the angular frequency is given by

$$
\omega^{2}=-\frac{64}{3} \frac{\left(\xi_{\sigma} F_{\lambda} Z_{\varphi}+2 F_{\sigma} F_{\varphi} \xi_{\varphi}-Z_{\varphi} F_{\sigma}^{2}-F_{\lambda} \xi_{\varphi}^{2}-\xi_{\sigma} F_{\varphi}^{2}\right) \dot{H}^{2}}{\left(F+4 H^{2} \dot{\xi}\right)\left[\left(F_{\lambda} Z_{\varphi}-F_{\varphi}^{2}\right)+8 H^{2}\left(F_{\sigma} Z_{\varphi}-F_{\varphi} \xi_{\varphi}\right)+16 H^{4}\left(\xi_{\sigma} Z_{\varphi}-\xi_{\varphi}^{2}\right)\right]} \frac{k^{4}}{a^{4}} .
$$

This case is, in a sense, discontinuous from the general case, as the degrees of freedom reduce by two, since the field $\delta \varphi_{M}$ becomes itself dependent on the field $\phi_{M}$. Eq. (52) implies that the $k^{4}$-term will be vanishing, for example, if $F=F(\varphi)$ and $\xi=\xi(\varphi)$.

\section{Case III: de Sitter $-\dot{H}=0$}

In this case, since both $\delta F$ and $\delta \xi$ are gauge invariant, we can not impose MGMG in general and (26) is not applicable anymore. Instead of using the variables involved in the MGMG, we find it convenient to use gauge invariant variables $\Phi$ defined by

$$
\Phi=-\frac{\delta F+4 H^{2} \delta \xi}{2 F_{0}}
$$

and $\delta \varphi$. Note that in de Sitter all background quantities except for the scale factor are constants. Using these perturbation variables, we find that the second order action can be written as

$$
\begin{aligned}
S^{(2)}=\frac{M_{P}^{2}}{16 \pi} \int \mathrm{d}^{3} x \mathrm{~d} t a^{3}[ & 6 F\left(\dot{\Phi}^{2}-a^{-2} \vec{\nabla} \Phi \cdot \vec{\nabla} \Phi\right)+\frac{8 \pi w}{M_{P}^{2}}\left(\dot{\delta} \dot{\varphi}^{2}-a^{-2} \vec{\nabla} \delta \varphi \cdot \vec{\nabla} \delta \varphi\right) \\
& \left.-m_{1}^{2} \Phi^{2}-m_{2}^{2} \Phi \delta \varphi-m_{3}^{2}(\delta \varphi)^{2}\right],
\end{aligned}
$$

where $m_{1}^{2}, m_{2}^{2}$ and $m_{3}^{2}$ are given by

$$
\begin{aligned}
m_{1}^{2} & =\frac{2 F\left(F-192 H^{6} \xi_{\sigma}-96 H^{4} F_{\sigma}-12 H^{2} F_{\lambda}\right)}{F_{\lambda}+8 H^{2} F_{\sigma}+16 H^{4} \xi_{\sigma}}, \\
m_{2}^{2} & =\frac{2 F\left(F_{\varphi}+4 H^{2} \xi_{\varphi}\right)}{F_{\lambda}+8 H^{2} F_{\sigma}+16 H^{4} \xi_{\sigma}} \\
m_{3}^{2} & =\frac{16 H^{4} \xi_{\varphi}^{2}-F_{\lambda} Z_{\varphi}+F_{\varphi}^{2}-16 H^{4} \xi_{\sigma} Z_{\varphi}+8 H^{2} F_{\varphi} \xi_{\varphi}-8 H^{2} Z_{\varphi} F_{\sigma}}{2\left(F_{\lambda}+8 H^{2} F_{\sigma}+16 H^{4} \xi_{\sigma}\right)} .
\end{aligned}
$$

We find that both fields propagate at the speed of light. In order not to have ghosts, the conditions $F>0$ and $w>0$ are required. 


\section{QUANTIZATION}

Up to here, we have considered only the classical theory of the perturbations. Although we have found non-trivial and interesting results at the classical level, nothing prevents us from going to quantize the perturbations. An obvious important application of the quantization will be the quantum generation of the curvature perturbations during inflation. In this case, inflation may be caused by the modification of gravity or by the scalar field $\varphi$ (inflaton). Then, the observed curvature perturbations will be a mixture of the scalar metric perturbations and the scalar field perturbations. Another possible application would be preheating [22, 23] after inflation, where the oscillations of $\varphi$ (if it is an inflaton) induces rapid creation of both $V_{1}$ and $V_{2}$ quanta. These applications are interesting by themselves and will be discussed elsewhere. In this section, we will provide general procedure to quantize the perturbations.

Our starting point is the second order action for the perturbations, which is given by Eq. (26). To quantize it, it is convenient to write the action in terms of canonical fields. Assuming $\operatorname{det} A \neq 0$, there always exists a time-dependent matrix $Z_{a b}(t)$ such that

$$
a^{3}(t) Z^{T} A Z=I,
$$

where $I$ is the identity matrix. Then the fields defined by $\psi_{a} \equiv\left(Z^{-1}\right)_{a b} V_{b}$ are the canonical fields, as their kinetic term becomes

$$
S^{(2)}=\int \mathrm{d} t \mathrm{~d}^{3} x \frac{1}{2} \delta_{a b} \dot{\psi}_{a} \dot{\psi}_{b}+\cdots
$$

However, as $Z$ depends on the time, we also get time derivative of $Z$ contributions coming from the first two terms in (26). This yields additional terms each of which has the same form as one of the last three terms in (26), which can be absorbed into a redefinition of the background dependent matrices. Therefore, without loss of generality, we can write the second order action like

$$
\begin{aligned}
S^{(2)}=\int \mathrm{d} t \mathrm{~d}^{3} x \mathcal{L} & \\
=\int \mathrm{d} t \mathrm{~d}^{3} x[ & \frac{1}{2} \delta_{a b} \dot{\psi}_{a} \dot{\psi}_{b}+b(t) \epsilon_{a b} \vec{\nabla} \dot{\psi}_{a} \cdot \vec{\nabla} \psi_{b}-\frac{1}{2} d_{a b}(t) \triangle \psi_{a} \triangle \psi_{b}-\frac{1}{2} H_{a b}(t) \vec{\nabla} \psi_{a} \cdot \vec{\nabla} \psi_{b} \\
& \left.+c(t) \epsilon_{a b} \dot{\psi}_{a} \psi_{b}-\frac{1}{2} m_{a b}(t) \psi_{a} \psi_{b}\right],
\end{aligned}
$$

where the background dependent matrices for each term are related to the matrices in (26) by $Z$. Although we can further perform an orthogonal transformation in order to make $d_{a b}(t)$ diagonal - still keeping the kinetic term diagonal - we leave it as a general symmetric matrix.

We can define canonical momenta conjugate to $\psi_{a}$ as

$$
\pi^{a}=\frac{\partial \mathcal{L}}{\partial \dot{\psi}_{a}}=\dot{\psi}_{a}-b \epsilon_{a b} \Delta \psi_{b}+c \epsilon_{a b} \psi_{b} .
$$

We impose the canonical quantization conditions, which are given by

$$
\begin{aligned}
& {\left[\hat{\psi}_{a}(t, \vec{x}), \hat{\psi}_{b}(t, \vec{y})\right]=\left[\hat{\pi}^{a}(t, \vec{x}), \hat{\pi}^{b}(t, \vec{y})\right]=0,} \\
& {\left[\hat{\psi}_{a}(t, \vec{x}), \hat{\pi}^{b}(t, \vec{y})\right]=i \delta_{a}^{b} \delta(\vec{x}-\vec{y}) .}
\end{aligned}
$$

The Heisenberg equations of motion, with the help of the above commutation relations, yield the following evolution equations for $\hat{\psi}_{a}$,

$$
\ddot{\hat{\psi}}_{a}-2 b \epsilon_{a b} \triangle \dot{\hat{\psi}}_{b}+2 c \epsilon_{a b} \dot{\hat{\psi}}_{b}+d_{a b} \triangle^{2} \hat{\psi}_{b}-\left(H_{a b}-\dot{b} \epsilon_{a b}\right) \triangle \hat{\psi}_{b}+\left(m_{a b}+\dot{c} \epsilon_{a b}\right) \hat{\psi}_{b}=0 .
$$

According to 24], it is always possible to write solutions of the equations consistent with the commutation relations as

$$
\hat{\psi}_{a}(t, \vec{x})=\int \frac{d^{3} k}{(2 \pi)^{3 / 2}}\left[U_{a b}(t, \vec{k}) \hat{a}_{b}(\vec{k}) e^{i \vec{k} \cdot \vec{x}}+U_{a b}^{*}(t, \vec{k}) \hat{a}_{b}^{\dagger}(\vec{k}) e^{-i \vec{k} \cdot \vec{x}}\right]
$$


Here, the time-independent operators $\hat{a}_{a}(\vec{k})$ satisfy the following commutation relations,

$$
\begin{aligned}
& {\left[\hat{a}_{a}\left(\vec{k}_{1}\right), \hat{a}_{b}\left(\vec{k}_{2}\right)\right]=\left[\hat{a}_{a}^{\dagger}\left(\vec{k}_{1}\right), \hat{a}_{b}^{\dagger}\left(\vec{k}_{2}\right)\right]=0,} \\
& {\left[\hat{a}_{a}\left(\vec{k}_{1}\right), \hat{a}_{b}^{\dagger}\left(\vec{k}_{2}\right)\right]=\delta_{a b} \delta\left(\vec{k}_{1}-\vec{k}_{2}\right) .}
\end{aligned}
$$

The matrix $U_{a b}(t, \vec{k})$ is a collection of c-number time-dependent functions which satisfy both the classical equation of motion,

$$
\ddot{U}_{a b}+2\left(b k^{2}+c\right) \epsilon_{a c} \dot{U}_{c b}+d_{a c} k^{4} U_{c b}+\left(H_{a c}-\dot{b}_{a c}\right) k^{2} U_{c b}+\left(m_{a c}+\dot{c} \epsilon_{a c}\right) U_{c b}=0,
$$

and the constraints given by

$$
\begin{aligned}
U U^{* T}-U^{*} U^{T} & =0, \\
\dot{U} \dot{U}^{* T}-\dot{U}^{*} \dot{U}^{T} & =-2 i\left(b k^{2}+c\right) \epsilon, \\
U \dot{U}^{* T}-U^{*} \dot{U}^{T} & =i I .
\end{aligned}
$$

These constraints are necessary for the consistency of the commutation relations (66), (67) with the canonical commutation relations (62), (63). By using the evolution equations (68), we can check that these constraints are satisfied at any time once they are imposed at one time. A simple example of $U$ and $\dot{U}$ that satisfy the constraints, is given by $U=I, \dot{U}=-\frac{i}{2} I-\left(b k^{2}+c\right) \epsilon$ at some initial time. However, there are infinite other combinations that satisfy the same constraints. Which $U$ we should use will depend on the physical situation (initial conditions) we are interested in. Here we do not specify the form of $U$.

As is well-known, there exists a state $|0\rangle$ which is defined by

$$
\hat{a}_{a}(\vec{k})|0\rangle=0 .
$$

Then we can construct a particle-basis by applying the creation operators $\hat{a}_{a}^{\dagger}(\vec{k})$ to the vacuum state. Once this is done, we can calculate expectation values of any operator for the state constructed in this way. For example, the "vacuum" two-point function is given by

$$
\left\langle 0\left|\hat{\psi}_{a}(t, \vec{x}) \hat{\psi}_{b}(t, \vec{y})\right| 0\right\rangle=\int \frac{\mathrm{d}^{3} k}{(2 \pi)^{3}} U_{a c}(t, \vec{k}) U_{b c}^{*}(t, \vec{k}) e^{i \vec{k} \cdot(\vec{x}-\vec{y})} .
$$

Before closing this section, let us finally make a comment. The evolution equations (64) in Fourier space can be seen as the equations of motion for an anisotropic charged harmonic oscillator with time-dependent spring force in a two-dimensional plane, in the presence of a time-dependent magnetic field. In fact, $\psi_{1}$ and $\psi_{2}$ represent the $x$ and $y$ coordinate of the point mass respectively, and the magnetic field whose strength is $2\left(b k^{2}+c\right)$ (for unit charge) is parallel to the $z$-axis. Therefore, the quantization of (60) is equivalent to the quantum mechanics of the harmonic oscillator under a time-dependent magnetic field. For time-independent spring forces in the presence of a time-independent magnetic field, 25, 26] gave the analytic wave function and energy spectrum, which, in terms of the Heisenberg picture we consider in this paper, means that analytic solutions of (68) were found. For the time-dependent case, 27, 28] found analytic wave function for an isotropic harmonic oscillator. For the general case, finding the analytic solution of (68) is not an easy task and it is beyond the analysis presented in this paper.

\section{CONCLUSION}

We have studied the scalar type cosmological perturbations for modified gravity models with a single scalar field. Followings are our findings.

- There are two dynamical perturbation variables, one from the gravity sector and one from the scalar field sector. As a result, we have four degrees of freedom. However, there are a few exceptions. In GR, as is well known, the scalar mode of gravity does not propagate and we have only one dynamical perturbation variable. Also the case $w=0$-no kinetic term for the scalar field - leads to only one propagating degree of freedom, which arises because gravity has been modified.

- Even in the presence of the scalar field, the action, expanded at second order in the fields, contains a term proportional to $k^{4}$. Therefore, inclusion of the matter field (at least in the form of a single scalar field) does 
not eliminate such term. To be more precise, among the four independent modes, two modes acquire a $k^{4}$ propagation. The propagation speed of these modes is affected by the presence of the scalar field through the modification of the background dynamics due to the scalar field. Depending on the modified gravity model and background solution, propagation becomes superluminal or the universe is unstable on small scales. The propagation speed of other two modes is equal to the speed of light.

- If the model satisfies the special condition $F_{\lambda} \xi_{\sigma}-F_{\sigma}^{2}=0$ and $w \neq 0$, the $k^{4}$-term disappears from the action.

- For the special case $F_{\lambda} \xi_{\sigma}-F_{\sigma}^{2}=0$, the propagation of the short wavelength modes are dominated by the $k^{2}$-term. If the combination $\dot{F} \xi_{\sigma}-\dot{\xi} F_{\sigma}$ does not vanish, none of the four modes propagates at the speed of light. It is very interesting and highly non-trivial that two modes which propagate at the speed of light in the general case no longer propagate at the speed of light in the special case.

- If the universe is de Sitter, irrespective of the MGM, all the modes propagate at the speed of light. Both $F$ and $w$ must be positive to avoid ghosts.

In Fig. 1 we summarize the classification of the MGM according to the properties of the propagation for the perturbation fields.

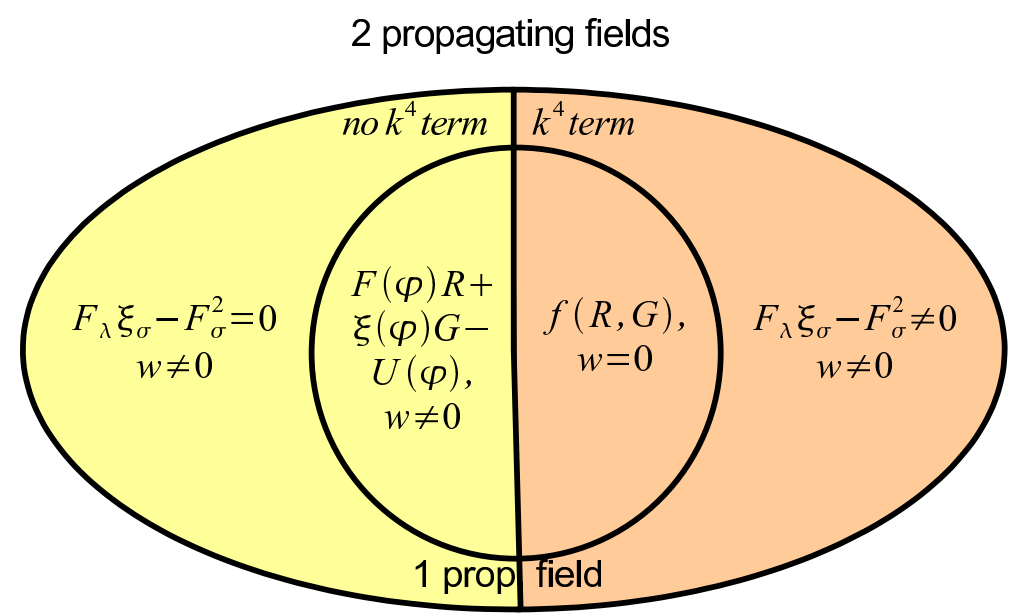

FIG. 1: Classification of the MGM according to the properties of the propagation for the perturbation fields. The models in the inner circle contain only one propagating field. The models in the orange (darker) region have modes which have the $k^{4}$-propagation, whereas those in the yellow (lighter) region do not have such modes.

\section{Acknowledgments}

We thank Jan Govaerts, Sean Murray, and Sebawe Abdalla for helpful discussions. This work is supported by the Belgian Federal Office for Scientific, Technical and Cultural Affairs through the Interuniversity Attraction Pole P6/11.

\section{APPENDIX A: MATRIX ELEMENTS $A_{a b}, D_{a b}$, AND $B$ FOR THE GENERAL CASE}

The matrix elements of $A_{a b}$ are given by

$$
A_{11}=\frac{2 a_{11}}{J}, \quad A_{12}=\frac{a_{12}}{J}, \quad A_{22}=\frac{8 \pi}{M_{P}^{2}} w \frac{a_{22}}{J},
$$


where

$$
\begin{aligned}
a_{11} & =(F+4 H \dot{\xi})\left(768 \xi_{\sigma} H^{8} \dot{\xi}^{2}+384 F_{\sigma} H^{6} \dot{\xi}^{2}+384 \xi_{\sigma} H^{6} \dot{F} \dot{\xi}+64 \xi_{\sigma} \dot{\varphi}^{2} \frac{16 \pi}{M_{P}^{2}} w \dot{\xi} H^{5}+48 F_{\lambda} \dot{\xi}^{2} H^{4}\right. \\
& +192 F_{\sigma} H^{4} \dot{F} \dot{\xi}+16 H^{4} F \xi_{\sigma} \dot{\varphi}^{2} \frac{16 \pi}{M_{P}^{2}} w+48 \xi_{\sigma} H^{4} \dot{F}^{2}+32 F_{\sigma} \dot{\varphi}^{2} \frac{16 \pi}{M_{P}^{2}} w \dot{\xi} H^{3}+24 F_{\lambda} \dot{F} \dot{\xi} H^{2} \\
& +8 H^{2} F F_{\sigma} \dot{\varphi}^{2} \frac{16 \pi}{M_{P}^{2}} w+192 F_{\sigma}^{2} \frac{16 \pi}{M_{P}^{2}} w \dot{\varphi}^{2} H^{2} \dot{H}^{2}-192 \frac{16 \pi}{M_{P}^{2}} w \dot{\varphi}^{2} \xi_{\sigma} F_{\lambda} H^{2} \dot{H}^{2}+24 F_{\sigma} H^{2} \dot{F}^{2} \\
& \left.+4 F_{\lambda} \dot{\varphi}^{2} \frac{16 \pi}{M_{P}^{2}} w \dot{\xi} H+3 F_{\lambda} \dot{F}^{2}+F F_{\lambda} \dot{\varphi}^{2} \frac{16 \pi}{M_{P}^{2}} w\right), \\
a_{12} & =\dot{\varphi} \frac{16 \pi}{M_{P}^{2}} w(F+4 H \dot{\xi})\left(192 \dot{\xi} \xi_{\sigma} H^{6}+96 \dot{\xi} F_{\sigma} H^{4}+12 \dot{\xi} F_{\lambda} H^{2}+32 \xi_{\sigma} F H^{5}+16 \xi_{\sigma} \dot{F} H^{4}\right. \\
& \left.+384 H^{3} F_{\sigma}^{2} \dot{H}^{2}+16 H^{3} F_{\sigma} F-384 H^{3} \xi_{\sigma} \dot{H}^{2} F_{\lambda}+8 F_{\sigma} \dot{F} H^{2}+2 F_{\lambda} F H+\dot{F} F_{\lambda}\right), \\
a_{22} & =768 H^{7} F \dot{\xi} \xi_{\sigma}+32 H^{3} F \dot{F} F_{\sigma}+384 H^{5} F \dot{\xi} F_{\sigma}+32 H^{4} F^{2} F_{\sigma}+64 H^{5} F \dot{F} \xi_{\sigma}+192 H^{4} \dot{\xi} F_{\sigma}+1152 H^{6} \dot{\xi}^{2} F_{\sigma} \\
& +384 H^{6} \dot{F} \dot{\xi} \xi_{\sigma}-3072 H^{5} \dot{H}^{2} \dot{\xi}\left(F_{\lambda} \xi_{\sigma}-F_{\sigma}^{2}\right)-768 H^{4} \dot{H}^{2} F\left(F_{\lambda} \xi_{\sigma}-F_{\sigma}^{2}\right)+\dot{F}^{2} F_{\lambda}+64 H^{6} F^{2} \xi_{\sigma}+8 H^{2} \dot{F}^{2} F_{\sigma} \\
& +4 H^{2} F^{2} F_{\lambda}+4 H F \dot{F} F_{\lambda}+144 H^{4} \dot{\xi}^{2} F_{\lambda}+16 H^{4} \dot{F}^{2} \xi_{\sigma}+2304 H^{8} \dot{\xi}^{2} \xi_{\sigma}+48 H^{3} F \dot{\xi} F_{\lambda}+24 H^{2} \dot{F} \dot{\xi} F_{\lambda},
\end{aligned}
$$

where $J$ is defined by

$$
\begin{aligned}
J & =768 H^{7} F \dot{\xi} \xi_{\sigma}+32 H^{3} F \dot{F} F_{\sigma}+384 H^{5} F \dot{\xi} F_{\sigma}+32 H^{4} F^{2} F_{\sigma}+64 H^{5} F \dot{F} \xi_{\sigma}+192 H^{4} \dot{F} \dot{\xi} F_{\sigma}+1152 H^{6} \dot{\xi}^{2} F_{\sigma} \\
& +384 H^{6} \dot{F} \dot{\xi} \xi_{\sigma}-3072 H^{5} \dot{H}^{2} \dot{\xi}\left(F_{\lambda} \xi_{\sigma}-F_{\sigma}^{2}\right)-64 H^{2} \dot{H}^{2} \frac{16 \pi}{M_{P}^{2}} w \dot{\varphi}^{2}\left(F_{\lambda} \xi_{\sigma}-F_{\sigma}^{2}\right)-768 H^{4} \dot{H}^{2} F\left(F_{\lambda} \xi_{\sigma}-F_{\sigma}^{2}\right)+\dot{F}^{2} F_{\lambda} \\
& +64 H^{6} F^{2} \xi_{\sigma}+8 H^{2} \dot{F}^{2} F_{\sigma}+4 H^{2} F^{2} F_{\lambda}+4 H F \dot{F} F_{\lambda}+144 H^{4} \dot{\xi}^{2} F_{\lambda}+16 H^{4} \dot{F}^{2} \xi_{\sigma}+2304 H^{8} \dot{\xi}^{2} \xi_{\sigma}+48 H^{3} F \dot{\xi} F_{\lambda} \\
& +24 H^{2} \dot{F} \dot{\xi} F_{\lambda} .
\end{aligned}
$$

Because $A_{a b}$ is symmetric matrix, $A_{21}=A_{12}$.

The quantity $B$ is given by

$$
B=-64 H \dot{H}^{2} \frac{16 \pi}{M_{P}^{2}} w \dot{\varphi}^{2}\left(\dot{F}+4 H^{2} \dot{\xi}\right) \frac{\left(F_{\lambda} \xi_{\sigma}-F_{\sigma}^{2}\right)}{J} .
$$

The matrix element of $D_{11}$ is given by

$$
D_{11}=-128 a \dot{H}^{2} \frac{\left(\dot{F}+4 H^{2} \dot{\xi}\right)^{2}\left(F_{\lambda} \xi_{\sigma}-F_{\sigma}^{2}\right)}{J},
$$

and all the other components, as already said, are identically equal to zero.

Except for $E_{22}, E_{a b}$ appears in the dispersion relation at the leading order only for the special case $F_{\lambda} \xi_{\sigma}-F_{\sigma}^{2}=0$. Therefore, we give their explicit expressions for the special case. Irrespective of modified gravity models, $E_{22}$ is given by (A10).

$$
\begin{gathered}
E_{11}=\frac{2}{a\left(\dot{F}+2 H F+12 H^{2} \dot{\xi}\right)^{2}}\left(256 H^{5} \dot{\xi}^{3}+256 H^{3} \dot{H} \dot{\xi}^{3}-64 H^{4} \dot{\xi}^{2} \ddot{\xi}+48 H^{4} F \dot{\xi}^{2}+128 H^{3} \dot{F}^{2}+16 H^{2} \frac{16 \pi}{M_{P}^{2}} w \dot{\varphi}^{2} \dot{\xi}^{2}\right. \\
+64 H^{2} \dot{H} F \dot{\xi}^{2}+64 H \dot{H} \dot{F}^{2}-32 H^{2} \dot{F} \dot{\xi} \ddot{\xi}+24 H^{2} F \dot{F} \dot{\xi}+8 H F \dot{\xi} \frac{16 \pi}{M_{P}^{2}} w \dot{\varphi}^{2}+16 H \dot{F}^{2} \dot{\xi} \\
\left.+16 \dot{H} F \dot{F} \dot{\xi}+3 F \dot{F}^{2}-4 \dot{F}^{2} \ddot{\xi}+\frac{16 \pi}{M_{P}^{2}} w \dot{\varphi}^{2} F^{2}\right), \\
E_{12}=\frac{1}{\left(\dot{F}+2 H F+12 H^{2} \dot{\xi}\right)\left(F_{\sigma}+4 H^{2} \xi_{\sigma}\right)}\left(-16 H^{3} \dot{\xi} \xi_{\sigma} \frac{16 \pi}{M_{P}^{2}} w \dot{\varphi}+32 H^{2} \dot{H} \dot{\xi} F_{\varphi} \xi_{\sigma}-32 H^{2} \dot{H} \dot{\xi} F_{\sigma} \xi_{\varphi}-4 H^{2} F \xi_{\sigma} \frac{16 \pi}{M_{P}^{2}} w \dot{\varphi}\right. \\
\left.\quad-4 H \dot{\xi} F_{\sigma} \frac{16 \pi}{M_{P}^{2}} w \dot{\varphi}+8 \dot{H} \dot{F} \xi_{\sigma} F_{\varphi}-8 \dot{H} \dot{F} F_{\sigma} \xi_{\varphi}-F F_{\sigma} \frac{16 \pi}{M_{P}^{2}} w \dot{\varphi}\right),
\end{gathered}
$$




\section{APPENDIX B: MATRIX ELEMENTS OF $A_{a b}, E_{a b}$ FOR THE SPECIAL CASE}

The matrix elements of $A_{a b}$ are given by

$$
\begin{aligned}
& A_{11}=\frac{2(F+4 H \dot{\xi})}{\left(\dot{F}+2 H F+12 H^{2} \dot{\xi}\right)^{2}}\left[3 \dot{F}^{2}+24 H^{2} \dot{F} \dot{\xi}+48 H^{4} \dot{\xi}^{2}+\frac{16 \pi}{M_{P}^{2}} w(F+4 H \dot{\xi}) \dot{\varphi}^{2}\right] \\
& A_{12}=-\frac{F+4 H \dot{\xi}}{\dot{F}+2 H F+12 H^{2} \dot{\xi}} \frac{16 \pi}{M_{P}^{2}} w \dot{\varphi} \\
& A_{22}=\frac{8 \pi w}{M_{P}^{2}}
\end{aligned}
$$

As for the matrix elements of $E_{a b}$, they are given by A8 , A9 and (A10).

We also give expressions for $\operatorname{det} A$, $\operatorname{det} E$ and $\operatorname{tr} A \operatorname{tr} E-\operatorname{tr}(A E)$, which are necessary for deriving the dispersion relation. They are given by

$$
\begin{aligned}
\operatorname{det} A & =\frac{3 \frac{16 \pi}{M_{P}^{2}} w(F+4 H \dot{\xi})\left(\dot{F}+4 H^{2} \dot{\xi}\right)^{2}}{\left(\dot{F}+2 H F+12 H^{2} \dot{\xi}\right)^{2}}, \\
\operatorname{tr} A \operatorname{tr} E & =\operatorname{tr}(A E)+\frac{2 \frac{16 \pi}{M_{P}^{2}} w\left(\dot{F}+4 H^{2} \dot{\xi}\right)^{2}}{\left(F_{\lambda}+4 H^{2} F_{\sigma}\right)\left(\dot{F}+2 H F+12 H^{2} \dot{\xi}\right)^{2}}\left(12 H^{2} F F_{\sigma}+56 H^{3} \dot{\xi} F_{\sigma}+14 H \dot{\xi} F_{\lambda}+8 \dot{H} F F_{\sigma}\right. \\
& \left.-8 H^{2} \ddot{\xi} F_{\sigma}-2 \ddot{\xi} F_{\lambda}+3 F F_{\lambda}+32 H \dot{H} \dot{\xi} F_{\sigma}\right), \\
\operatorname{det} E & =-\frac{\left(\dot{F}+4 H^{2} \dot{\xi}\right)^{2}}{\left(\dot{F}+2 H F+12 H^{2} \dot{\xi}\right)^{2}\left(F_{\lambda}+4 H^{2} F_{\sigma}\right)^{2} \dot{\varphi}^{2}}\left(64 \dot{H}^{2} \dot{\xi}^{2} F_{\lambda}^{2}-256 H^{3}\left(H^{2}+\dot{H}\right) \dot{\xi} F_{\sigma}^{2} \frac{16 \pi}{M_{P}^{2}} w \dot{\varphi}^{2}\right. \\
& -128 H^{3} \dot{\xi} F_{\sigma} F_{\lambda} \frac{16 \pi}{M_{P}^{2}} w \dot{\varphi}^{2}-64 H \dot{H} \dot{\xi} F_{\lambda} F_{\sigma} \frac{16 \pi}{M_{P}^{2}} w \dot{\varphi}^{2}-16 H \dot{\xi} F_{\lambda}^{2} \frac{16 \pi}{M_{P}^{2}} w \dot{\varphi}^{2}-128 \dot{H}^{2} \dot{F} \dot{\xi} F_{\lambda} F_{\sigma} \\
& +64 H^{4} \ddot{\xi} F_{\sigma}^{2} \frac{16 \pi}{M_{P}^{2}} w \dot{\varphi}^{2}-48 H^{4} F F_{\sigma}^{2} \frac{16 \pi}{M_{P}^{2}} w \dot{\varphi}^{2}+32 H^{2} \ddot{\xi}_{\sigma} F_{\lambda} \frac{16 \pi}{M_{P}^{2}} w \dot{\varphi}^{2}-24 H^{2} F F_{\lambda} F_{\sigma} \frac{16 \pi}{M_{P}^{2}} w \dot{\varphi}^{2} \\
& \left.-64 H^{2} \dot{H} F F_{\sigma}^{2} \frac{16 \pi}{M_{P}^{2}} w \dot{\varphi}^{2}+64 \dot{H}^{2} \dot{F}^{2} F_{\sigma}^{2}-3 F F_{\lambda}^{2} \frac{16 \pi}{M_{P}^{2}} w \dot{\varphi}^{2}-16 \dot{H} F F_{\lambda} F_{\sigma} \frac{16 \pi}{M_{P}^{2}} w \dot{\varphi}^{2}+4 \ddot{\xi} F_{\lambda}^{2} \frac{16 \pi}{M_{P}^{2}} w \dot{\varphi}^{2}\right) .
\end{aligned}
$$

[1] A. De Felice and T. Suyama, JCAP 0906, 034 (2009), 0904.2092.

[2] S. M. Carroll, A. De Felice, V. Duvvuri, D. A. Easson, M. Trodden, and M. S. Turner, Phys. Rev. D 71, 063513 (2005), arXiv:astro-ph/0410031.

[3] A. Nunez and S. Solganik, Phys. Lett. B608, 189 (2005), hep-th/0411102.

[4] S. Nojiri, S. D. Odintsov, and P. V. Tretyakov, Phys. Lett. B651, 224 (2007), 0704.2520.

[5] I. Navarro and K. Van Acoleyen, Phys. Lett. B622, 1 (2005), gr-qc/0506096.

[6] I. Navarro and K. Van Acoleyen, JCAP 0603, 008 (2006), gr-qc/0511045.

[7] O. Mena, J. Santiago, and J. Weller, Phys. Rev. Lett. 96, 041103 (2006), astro-ph/0510453.

[8] A. De Felice, M. Hindmarsh, and M. Trodden, JCAP 0608, 005 (2006), astro-ph/0604154.

[9] C. Bonvin, C. Caprini, and R. Durrer, Phys. Rev. Lett. 97, 081303 (2006), astro-ph/0606584.

[10] A. Hashimoto and N. Itzhaki, Phys. Rev. D63, 126004 (2001), hep-th/0012093.

[11] J.-P. Bruneton, Phys. Rev. D75, 085013 (2007), gr-qc/0607055.

[12] G. Ellis, R. Maartens, and M. A. H. MacCallum, Gen. Rel. Grav. 39, 1651 (2007), gr-qc/0703121.

[13] E. Babichev, V. Mukhanov, and A. Vikman, JHEP 02, 101 (2008), 0708.0561.

[14] A. A. Starobinsky, Phys. Lett. B91, 99 (1980).

[15] S. Capozziello, Int. J. Mod. Phys. D11, 483 (2002), gr-qc/0201033.

[16] S. M. Carroll, V. Duvvuri, M. Trodden, and M. S. Turner, Phys. Rev. D70, 043528 (2004), astro-ph/0306438.

[17] C. Brans and R. H. Dicke, Phys. Rev. 124, 925 (1961).

[18] R. H. Dicke, Phys. Rev. 125, 2163 (1962).

[19] J.-c. Hwang and H. Noh, Phys. Rev. D71, 063536 (2005), gr-qc/0412126.

[20] A. De Felice and S. Tsujikawa, Phys. Lett. B675, 1 (2009), 0810.5712.

[21] A. De Felice and S. Tsujikawa (2009), 0907.1830. 
[22] J. H. Traschen and R. H. Brandenberger, Phys. Rev. D42, 2491 (1990).

[23] L. Kofman, A. D. Linde, and A. A. Starobinsky, Phys. Rev. Lett. 73, 3195 (1994), hep-th/9405187.

[24] S. Groot Nibbelink and B. J. W. van Tent, Class. Quant. Grav. 19, 613 (2002), hep-ph/0107272.

[25] T. K. Rebane, Theoretical and Experimental Chemistry 5, 1 (1969).

[26] I. M. Ternov, V. G. Bagrov, and V. N. Zadorozhnyi, Soviet Physics Journal 14, 492 (1971).

[27] M. S. Abdalla, Phys. Rev. A 37, 4026 (1988).

[28] M. S. Abdalla, Nuovo Cim. B 101, 267 (1988). 